Proceedings of the ASME 2017 36th International Conference on Ocean, Offshore and Arctic Engineering
OMAE2017
June 25-30, 2017, Trondheim, Norway

\title{
A STUDY OF 3D FLEXIBLE CAUDAL FIN FOR FISH PROPULSION
}

\author{
Guangyu Shi \\ Department of Naval Architecture, Ocean and \\ Marine Engineering, University of Strathclyde \\ Glasgow, G4 OLZ, UK
}

\author{
Qing Xiao \\ Department of Naval Architecture, Ocean and \\ Marine Engineering, University of Strathclyde \\ Glasgow, G4 OLZ, UK
}

\author{
Qiang Zhu \\ Department of Structural Engineering, \\ University of California San Diego \\ La Jolla, CA 92093, USA
}

\section{ABSTRACT}

This paper is inspired by a recent numerical study (Shoele and Zhu, 2012, "Leading edge strengthening and the propulsion performance of flexible ray fins," Journal of Fluid Mechanics, Vol. 693, pp. 402-432), which shows that, for a 2D flexible ray replicating the pectoral fins of live fish, undergoing a flapping motion in a viscous fluid, the performance can be significantly improved via the flexibility distribution on the rays. In present study, we investigate the propulsion capability of a 3D caudal fin undergoing a flapping motion. The embedded rays are modeled as linear springs and the soft membrane is modeled as a flexible plate being able to deform in span-wise direction. A finite-volume method based NavierStokes solver is used to solve the fluid-structure interaction problem. The present paper focuses on the effects of various distributions of the ray and the ray flexibilities, which can lead to different fin deformations. It is shown that the detailed ray distribution has significant influence on the propulsion performance. By distributing fin rays at the tips rather than the middle of fin, a less power expenditure is observed, leading to higher propulsion efficiency. However, larger thrust force is obtained through distributing the rays at the middle, which is attributed to larger effective flapping amplitude. Additionally, ray flexibilities also play a pivotal role in the thrust generation of the fin.

\section{INTRODUCTION}

Fishes rely on the undulation of their flexible bodies and/or the flapping of different fins for locomotion and maneuvering.
For most bony fishes, the fins can be characterized as a soft and thin membrane reinforced by skeleton rays. The stiffness of the membrane is much smaller than the bony rays, i.e. the rigidity of the fin is mainly determined by the embedded fin rays. By individually actuating the muscles at the basal end of each ray, the fish fin can be actively controlled to achieve higher stability and maneuverability.

Due to the potential applications in biomimetic engineering, the ray fins have attracted much attention. Experimentally, Tangorra et al. (2007, 2010) studied the hydrodynamic performance and fin ray flexibility effects by mechanically imitating the motion of the bluegill sunfish pectoral fin. Zhu and Shoele (2008) computationally examined a trapezoid caudal fin strengthened by a number of nonlinear beams which resembled the embedded rays. They found that deformability of caudal fins greatly enhanced the propulsion efficiency, and it also reduced the sensitivity of efficiency to the kinematic parameters. Shoele and Zhu (2009, 2010) numerically analyzed the performance of a skeletonstrengthened pectoral fin by a potential flow assumption. They concluded that passive deformation of the ray fins could increase the thrust and propulsion efficiency and emphasized the influence of kinematic parameters and phase lag between rays. Considering the viscous effects, Shoele and Zhu (2012) studied a two-dimensional flexible ray fin with strengthened leading edge. In their model, the fin was modeled as a flexible but inextensible membrane, and the underlying rays were represented by springs. By comparing with the rigid rays, they found that both the thrust production and propulsion efficiency can be significantly improved via the flexibility distribution on the rays. 
(A)

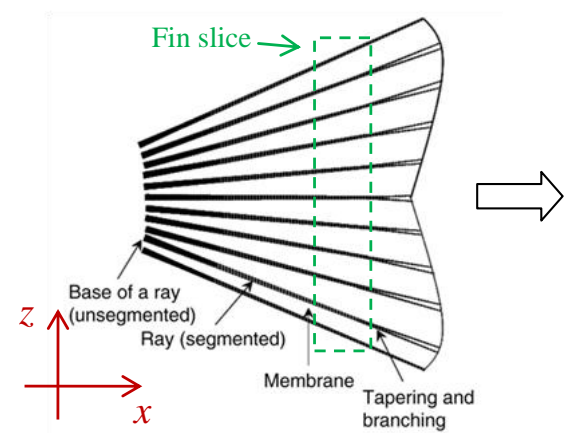

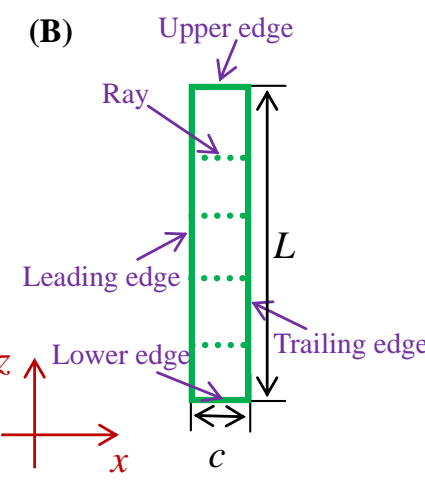

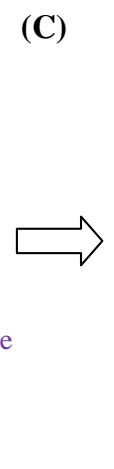

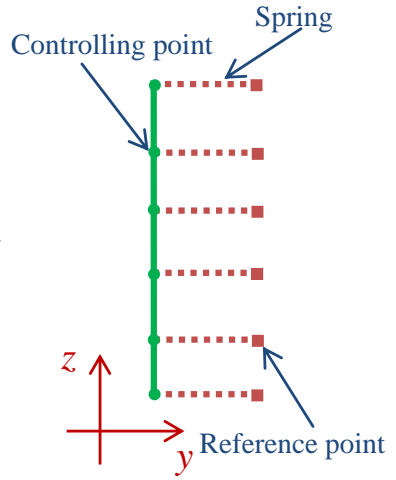

Fig. 1 (a) Schematic illustration of a typical caudal fin (Zhu and Shoele 2008). (b) Geometrically and structurally simplified rectangular fin used in present study. (c) Model representation of the fin ray.

However, because a 2D study was conducted, in which the flow at a chord-wise cross section of a pectoral fin is considered, the model is too idealized to reflect the realistic fish fins, in which span-wise deformation is equally important .

To investigate the effects of span-wise deformation on the performance of a caudal fin, the current study is based on a 3D passively span-wise deformable fin through a fully coupled fluid-structure interaction numerical modeling. Following Shoele and Zhu (2012), the fin is modeled as a flexural rectangular wing with small rigidity and the rays are represented by springs. The investigation is carried out using a finite volume based in-house CFD code coupled with a nonlinear beam model. The main objective of this study is to examine how the span-wise deformations influence the propulsion performance of caudal fin.

\section{PROBLEM STATEMENT}

Figure 1 (a) shows the caudal fin of a bluegill sunfish, which is geometrically composed of a soft membrane and several skeleton rays. Instead of investigating such a complex geometry, we study only a slice of this fin and further idealize it as a rectangular fin, as shown in Fig. 1 (b). This rectangular fin has a chord length of $c$ and an aspect ratio of 6 , and the thickness is $h=0.05 c$, which is assumed to be uniform except for the tapering at all edges. The fin is modeled as a flexural but inextensible plate, which is geometrically threedimensional. However, the fin is only allowed to deform in $y-z$ plane, which is essentially two-dimensional. Considering this, the deformation of the fin can be represented by the distortion of its leading edge, which can be modeled as a flexible but inextensible beam. The motion of the fin is controlled by $N$ controlling points distributed along the beam which represent the actual positions of fin rays, as illustrated in Fig. 1 (c). For each controlling point, there is a corresponding reference point with prescribed motion, which depicts the trajectory of a ray if it is rigid. The flexibility of the ray is represented by a linear spring connecting each pair of controlling and reference points.

The flapping motion of the fin is actuated by the heave motion of the reference points. For the $i$ th reference point, the heaving motion can be prescribed as $y_{i}(t)=a_{0} \sin \left(\omega t+\varphi_{i}\right)$, where $a_{0}$ is the heaving amplitude, $\omega$ is the frequency, $\varphi_{i}$ is the phase and $t$ is the time. The Strouhal number which characterizes the unsteady fluid dynamics is defined based on the peak-to-peak amplitude of the reference points as $S_{t}=2 a_{0} f / U_{0}$, where $f=1 / T=2 \pi / \omega$ is the heaving frequency. The Reynolds number is defined based on chord length as $R e=\rho U_{0} c / \mu$. In the present study, we select $a_{0}=0.5 c, \varphi_{i}=0,(i=1, N), S_{t}=0.4$ and $R e=300$.

\section{MATHEMATICAL FORMULATIONS AND NUMERICAL METHODS}

\section{Fluid Dynamics Around the Flexible Fin}

The dynamics of Newtonian flow is governed by the conservation laws of mass, momentum and energy. Under the assumption of no internal heat or mass sources, and by neglecting body forces, the integral governing equations can be written as

$$
\frac{\partial}{\partial t} \iiint_{\Omega} \mathbf{W} d V+\iint_{\partial \Omega} \mathbf{F}_{c} \cdot \mathbf{n} d S-\iint_{\partial \Omega} \mathbf{F}_{d} \cdot \mathbf{n} d S=0,
$$

where $\Omega$ is the control volume and $\partial \Omega$ is the closed boundary surface, and $\mathbf{n}$ is the surface unit normal vector. In Eq.(1), the conservative variable vector is defined as

$$
W=\{\rho, \rho \mathbf{u}, \rho E\}^{T},
$$

where $\rho$ is the fluid density, $\mathbf{u}=\{u, v, w\}$ is the velocity vector in Cartesian coordinate system, and $E$ is the total energy of the flow. In Eq.(1), $\mathbf{F}_{c}$ and $\mathbf{F}_{d}$ are flux tensors representing the convective and diffusive fluxes respectively. The convective fluxes expressed in terms of the contravariant velocity are

$$
\mathbf{F}_{c}=\left[\begin{array}{c}
\rho \hat{\mathbf{u}}^{T} \\
\rho \hat{\mathbf{u}}+p \mathbf{I} \\
(\rho E \hat{\mathbf{u}}+p \mathbf{u})^{T}
\end{array}\right],
$$


where $\hat{\mathbf{u}}=(\hat{u}, \hat{v}, \hat{w})^{T}$ is the relative velocity which can be calculated as $\hat{\mathbf{u}}=\mathbf{u}-\mathbf{u}_{g}$, and $\mathbf{u}_{g}=\left(u_{g}, v_{g}, w_{g}\right)^{T}$ is the grid velocity vector. The diffusive fluxes caused by the viscous shear stresses and thermal conduction are

$$
\mathbf{F}_{d}=\left[\begin{array}{c}
0 \\
\boldsymbol{\tau} \\
(\boldsymbol{\tau} \cdot \mathbf{u}-\mathbf{q})^{T}
\end{array}\right],
$$

where the shear stress and heat flux are expressed as

$$
\begin{aligned}
& \tau_{i j}=\mu\left[\left(\frac{\partial u_{i}}{\partial x_{j}}+\frac{\partial u_{j}}{\partial x_{i}}\right)-\frac{2}{3}(\nabla \cdot \mathbf{u}) \delta_{i j}\right], \\
& \mathbf{q}=-\kappa \nabla T
\end{aligned}
$$

where $\mu$ is the laminar dynamic viscosity which can be evaluated using Sutherland's Formula, $\kappa$ is the laminar thermal conductivity, and $T$ is the temperature.

Following Liu and Zheng (1996) and Liu and Ji (1996), a cell-centred finite volume method based on a multi-block structured grid is used. For each cell $(i, j, k)$, the semi-discrete form of the governing equations can be rewritten as

$$
\frac{\partial}{\partial t}(\mathbf{W} \Delta V)_{i, j, k}-\mathbf{R}_{i, j, k}=0,
$$

where $\mathbf{R}_{i, j, k}$ is the residual, which arises from the discretised convective and diffusive terms. In present code, the convective term is discretised by the central JST scheme with artificial dissipation proposed by Jameson et al. (1981), and the first order derivatives are obtained using Green's theorem.

The temporal term is discretised by an implicit backward difference scheme with second order accuracy, and by adopting the dual-time stepping algorithm proposed by Jameson (1991), Eq.(6) can be reformulated as a steady-state problem with a pseudo-time

$$
\frac{\partial}{\partial t^{*}} \mathbf{W}^{n+1}=\frac{1}{\Delta V^{n+1}} \mathbf{R}^{*}\left(\mathbf{W}^{n+1}\right),
$$

where

$$
\begin{aligned}
\mathbf{R}^{*}\left(\mathbf{W}^{n+1}\right)= & \mathbf{R}\left(\mathbf{W}^{n+1}\right)- \\
& \frac{3(\mathbf{W} \Delta V)^{n+1}-4(\mathbf{W} \Delta V)^{n}+(\mathbf{W} \Delta V)^{n-1}}{2 \Delta t} .
\end{aligned}
$$

The semi-discrete Eq.(7) is integrated using a hybrid multistage Runge-Kutta scheme. Besides, local time stepping, residual smoothing and multigrid techniques are adopted in order to increase the stability and accelerate the convergence of the solution. MPI is used for exchanging information between different CPUs in parallel computation.

\section{Structural Dynamics of the Flexible Fin}

The flexible fin is modelled as a two-dimensional thin plate with uniform thickness h and Young's modulus E. Using thin-body assumptions $(h<<L)$ and employing variational analysis, the structural dynamics is governed by

$$
\begin{aligned}
& m_{s} \frac{\partial^{2} \mathbf{x}}{\partial t^{2}}+K_{b} \frac{\partial^{4} \mathbf{x}}{\partial s^{4}}-K_{h} \frac{\partial}{\partial s}\left\{\left[1-\left(\frac{\partial \mathbf{x}}{\partial s} \cdot \frac{\partial \mathbf{x}}{\partial s}\right)^{-1 / 2}\right] \frac{\partial \mathbf{x}}{\partial s}\right\} \\
& =\mathbf{F}_{f}+\mathbf{F}_{e}, 0<s<L,
\end{aligned}
$$

where $m_{s}=\rho_{s} h$ is the mass per unit length of the fin, $K_{b}=E h^{3} / 12$ is the bending rigidity and $K_{h}=E h$ represents the stretching rigidity. In Eq.(9), the inertia, elastic bending and stretching effects are depicted by the first, second and third term on the left-hand side respectively. On the other side, $\mathbf{F}_{f}$ is the fluid loads which are calculated by evaluating the difference between the stress tensors (including pressure) at the top and bottom of the body. $\mathbf{F}_{e}$ is the force imposed by the controlling points of the fin. It is evaluated as $\mathbf{F}_{e}=\sum_{i=1}^{N} \mathbf{F}_{e, i} \delta\left(s-s_{i}\right)$, where $s_{i}$ is the position of the $i$ th controlling point. The connection between the $i$ th reference point and the corresponding controlling point is modelled as a linear spring, so the connecting force can be evaluated as $\mathbf{F}_{e, i}=k_{i}\left(\mathbf{x}_{r, i}-\mathbf{x}_{c, i}\right), i=1, N$, where $k_{i}$ is the spring stiffness, which represents the rigidity of the ray. $\mathbf{x}_{r, i}$ is the position of the $i$ th reference point and $\mathbf{x}_{r, i}$ is the position of the $i$ th controlling point. To model the damping effect due to the internal friction, the Young's modulus $E$ in Eq.(9) is replaced by $E(1+\mathrm{G} \partial / \partial \mathrm{t})$, where $G$ denotes the energy dissipation magnitude.

At both ends of the structural model, free boundary condition (zero-stress and zero-bending) is applied. It can be mathematically expressed as

$$
\begin{gathered}
-E h\left[1-\left(\frac{\partial \mathbf{x}}{\partial s} \cdot \frac{\partial \mathbf{x}}{\partial s}\right)^{-1 / 2}\right] \frac{\partial \mathbf{x}}{\partial s}+\frac{E h^{3}}{12} \frac{\partial^{3} \mathbf{x}}{\partial s^{3}}=0, \\
\frac{\partial^{2} \mathbf{x}}{\partial s^{2}}=0,
\end{gathered}
$$

The governing equation and the boundary condition equations are numerically discretised using a finite difference method. The resulting linear equations are solved by adopting a Newtonian iterative solver.

\section{Fluid-Structure Coupling}

Generally, the coupled fluid-structure system can be resolved either by monolithic or partitioned approaches. The monolithic method is both mathematically and computationally challenging, while in the partitioned approach, the flow and structural equations are solved separately, and the coupling between the two solvers are only limited to the fluid-structure interface. It is relatively simple to implement and less changes in the existing codes are needed. Therefore, a partitioned 
approach referred as Conventional Serial Staggered (CSS) procedure (Farhat and Lesoinne 2000) is adopted in present work.

Because the fluid equations and structural model are solved independently, the structural grid does not necessarily coincide with the body-fitted flow mesh. Thus, the interpolations of fluid forces and structural deformations must be performed between the two grid systems. The flow grid displacements $\Delta \mathbf{x}_{F}$ can be expressed in terms of structural grid displacements $\Delta \mathbf{x}_{s}$ using a transformation matrix $\mathbf{P}$ as

$$
\Delta \mathbf{x}_{F}=\mathbf{P} \Delta \mathbf{x}_{S} .
$$

Considering the requirement of conservativeness, the corresponding matrix for force transformation can be derived as

$$
\begin{gathered}
\mathbf{F}_{S}^{T} \Delta \mathbf{x}_{S}=\mathbf{F}_{F}^{T} \Delta \mathbf{x}_{F}=\mathbf{F}_{F}^{T} \mathbf{P} \Delta \mathbf{x}_{S}, \\
\mathbf{F}_{S}=\mathbf{P}^{T} \mathbf{F}_{F},
\end{gathered}
$$

where $\mathbf{F}_{S}$ is the force vector imposed on the structural mesh and $\mathbf{F}_{F}$ represents the force vector imposed on the fluid mesh. The transformation matrix $\mathbf{P}$ is obtained using the Constant Volume Tetrahedron (CVT) method (Goura et al. 2001).

\section{Performance Evaluation}

To measure the performance of the caudal fin, the instantaneous input power is evaluated as

$$
P=\sum_{i=1}^{N} \mathbf{F}_{e, i} \cdot \dot{\mathbf{x}}_{r, \mathrm{i}}
$$

Through integrating the fluid forces along $-x$ direction, the thrust force generated by the fin can be calculated as

$$
F_{T}=-\int_{\Gamma} F_{x}(s, t) d s
$$

where $\Gamma$ denotes the contour of the fin and $F_{x}$ is the $x$ component of $\mathbf{F}_{f}$. The corresponding power coefficient and thrust coefficient can be written as

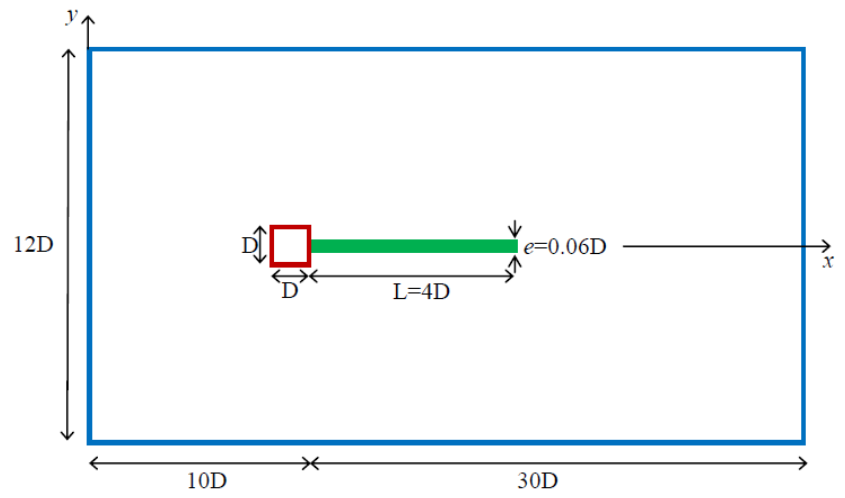

Fig. 2 Diagram of a flexible cantilever attached behind a square cylinder

$$
\begin{aligned}
& C_{P}=\frac{P}{1 / 2 \rho U_{0}^{3} S_{0}}, \\
& C_{T}=\frac{F_{T}}{1 / 2 \rho U_{0}^{2} S_{0}},
\end{aligned}
$$

where $S_{0}$ is the reference area.

The efficiency is defined as

$$
\eta=\frac{\bar{C}_{T}}{\bar{C}_{P}},
$$

where $\bar{C}_{T}$ and $\bar{C}_{P}$ are the time-averaged values of $C_{T}$ and $C_{P}$ respectively.

\section{VALIDATIONS}

The flow solver used in present study has been extensively validated in previous publications of our group (Xiao and Liao 2010; Xiao et al., 2012; Liu et al. 2013, 2016). In present work, the flow solver and the coupling with a nonlinear beam model will be further validated.

\section{Flow induced vibrations a flexible cantilever}

This case has been widely used for the validation of fluidstructure interaction solvers by previous researchers (Matthies and Steindorf 2003; Dettmer and Peric 2006; Wood et al. 2010; Kassiotis et al. 2011; Habchi et al. 2013) as a benchmark. As shown in Fig. 2, a thin elastic cantilever is placed in the wake of a fixed rigid square cylinder. The diameter of the square cylinder is $D$, and the length and thickness of the cantilever is $L=4 D$ and $e=0.06 D$ respectively. The top and bottom boundaries are considered as symmetry planes. At the inlet and outlet, the far-field boundary conditions are applied. Since the compressible Navier-Stokes equations are solved in present solver, the computational domain as well as inlet and outlet boundary conditions are different from referred literatures. The Reynolds number based on $D$ is $R e=333$.

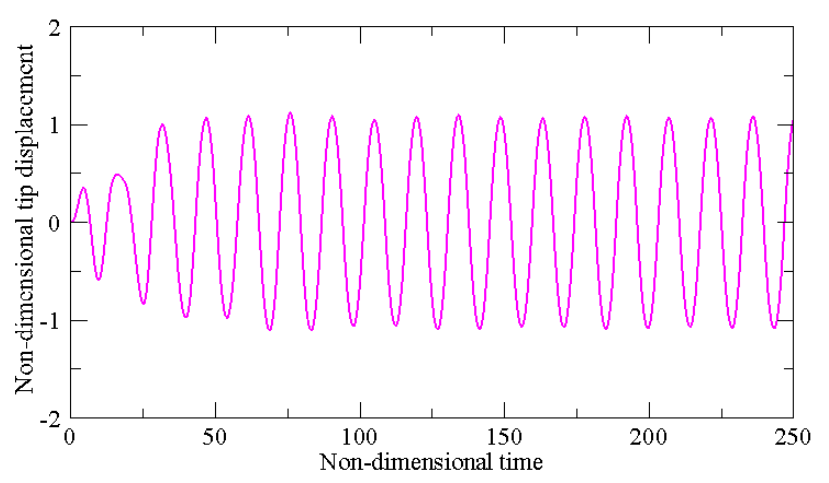

Fig. 3 Tip displacement as a function of non-dimensional time 


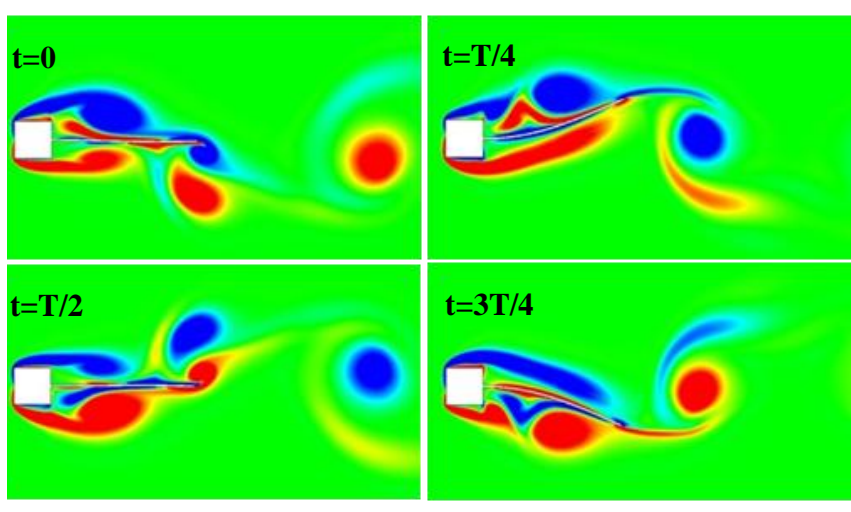

Fig. 4 Flow vorticity when tip is at extreme positions

Table 1 Comparison of present results with those in literatures

\begin{tabular}{|l|c|c|}
\hline Author & $f^{*}$ & $d_{\max }^{*}$ \\
\hline Matthies et al., 2003 & 0.192 & 1.18 \\
\hline Dettmer et al., 2006 & 0.185 & 1.25 \\
\hline Wood et al., 2010 & 0.179 & 1.15 \\
\hline Kassiotis et al., 2011 & 0.182 & 1.05 \\
\hline Habchi et al., 2013 & 0.201 & 1.02 \\
\hline Present study & 0.213 & 1.08 \\
\hline
\end{tabular}

Figure 3 demonstrates the time history of cantilever tip displacement. The non-dimensional time $t^{*}$ and the nondimensional displacement $d^{*}$ are defined as $t^{*}=t U / D$ and $d^{*}=d / D$ respectively. The vibration of the cantilever becomes periodic after a transient region. Fig. 4 represents vorticity contours at extreme positions. It can be observed that flow separates at the leading corners and a clockwise vortex forms at the upper region while its counterpart forms at the lower region. These vortices travel along the vibrating cantilever and impinge with the vortices formed at the trailing edge and then dissipate in the wake. A closer observation also reveals that a clockwise vortex forms at the trailing edge during the upward motion of the cantilever while the counter-clockwise one forms when the cantilever moves downwards. The vortices at the trailing edge are shed into the wake forming the famous Von Karman vortex street. The reduced frequency $\left(f^{*}=\pi f D / U\right)$ and the dimensionless maximal tip displacement $\left(d_{\max }^{*}\right)$ are summarised in Table 1. Obviously, the predicted results in present study agree very well with previous results. The reduced frequency from present simulation is slightly higher than the results from referred literatures, but it still lie in a reasonable region. The maximal tip displacement obtained here
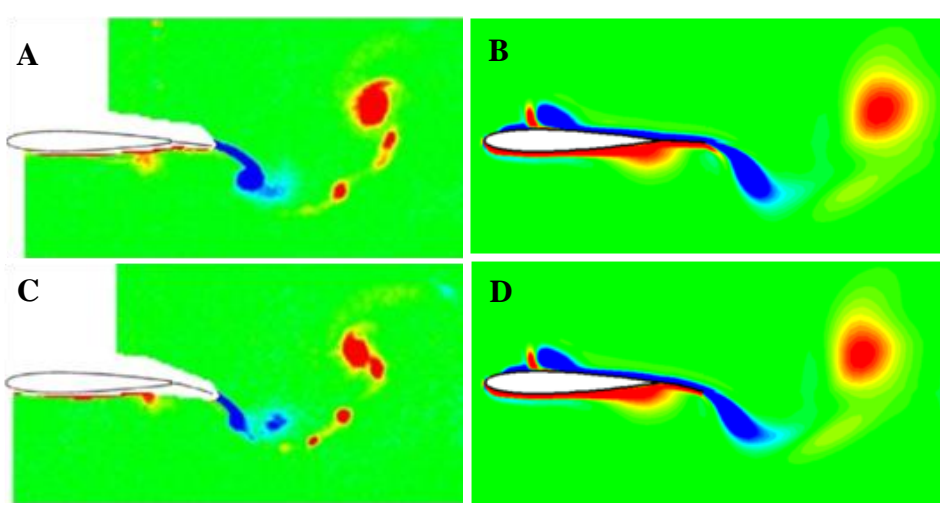

Fig. 5 Flow vorticity at $t=3 T / 4, S_{t}=0.16$, experiment $(a, c)$, present simulation $(b, d)$, flexible case $(a, b)$, highly flexible case $(c, d)$.

$d_{\max }^{*}=1.08$ is close to those obtained using different FSI solvers, which ranges from 1.02 to 1.25 .

\section{Plunging foil with a flexible trailing edge}

Present FSI solver is further validated by simulating a plunging foil with an elastic trailing edge. The computed results are compared with the experimental data by Cleaver et al. (2014). Fig. 5 demonstrates flow vorticity contours when the foil is in the middle and moving upwards. As we can observe that for all cases the flow vorticity contour are qualitatively similar to the conclusion drawn by Cleaver et al. (2014). In fact, a counter-clockwise TEV forms when the foil moves downward and its clockwise counterpart forms during the upward motion. All these TEVs convect into the wake and form the well-known reversed Von Karman vortex sheet. Fig. 6 shows the amplitude ratio as a function of Strouhal number based on chord length. The numerical results and experimental data are in good agreement for both optimal and post-optimal cases.

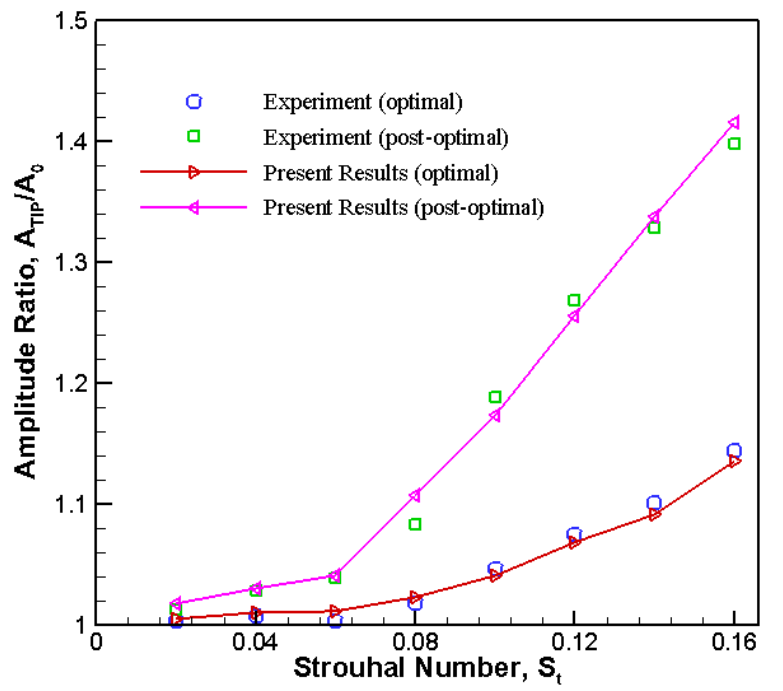

Fig. 6 Amplitude ratio as a function of Strouhal number 
Fin I

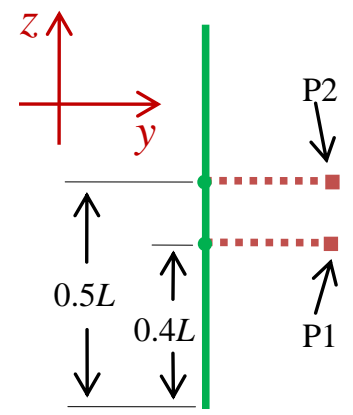

Fin II

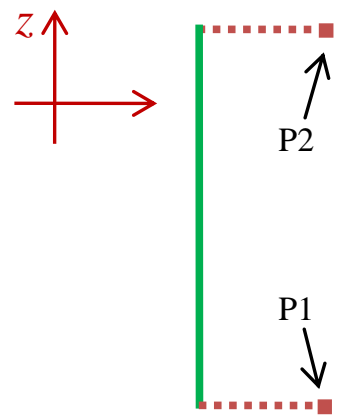

Fin III

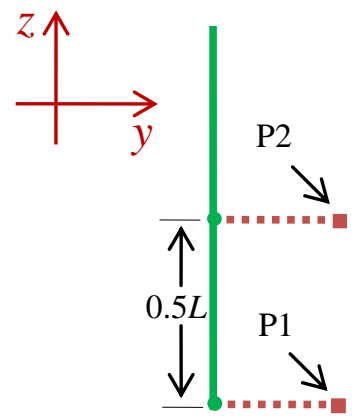

Fig. 7 Diagrams of three different distributions of fin rays.

Table 2. Summary of mean thrust, power input coefficients and efficiency

\begin{tabular}{|l|c|c|c|c|c|c|}
\hline & \multicolumn{2}{|c|}{ fin I } & \multicolumn{2}{c|}{ fin II } & \multicolumn{2}{c|}{ fin III } \\
\hline & flexible & highly flexible & flexible & highly flexible & flexible & highly flexible \\
\hline $\bar{C}_{T}$ & 0.0606 & 0.0672 & 0.0274 & 0.0336 & 0.0483 & -0.0003 \\
\hline $\bar{C}_{P}$ & 0.4044 & 0.4644 & 0.1416 & 0.1517 & 0.3853 & 0.1984 \\
\hline$\eta$ & 0.1498 & 0.1447 & 0.1936 & 0.2217 & 0.1253 & -0.0015 \\
\hline
\end{tabular}

(A)

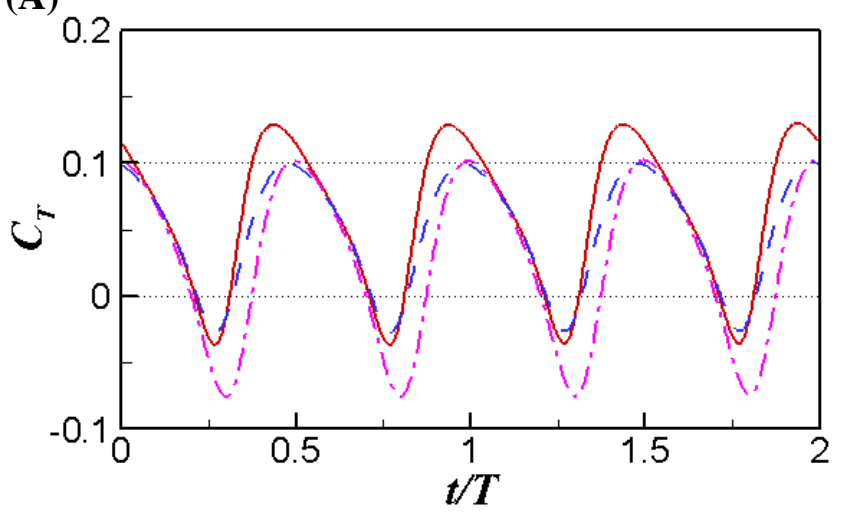

(C)

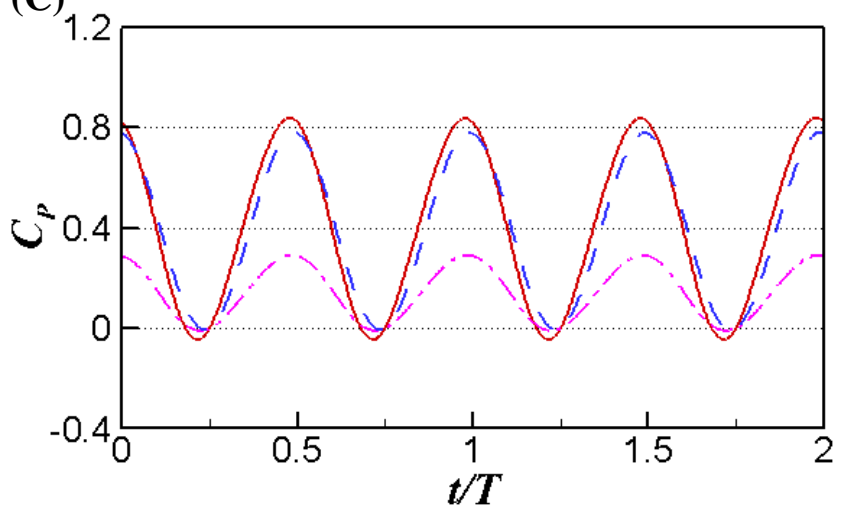

(B)

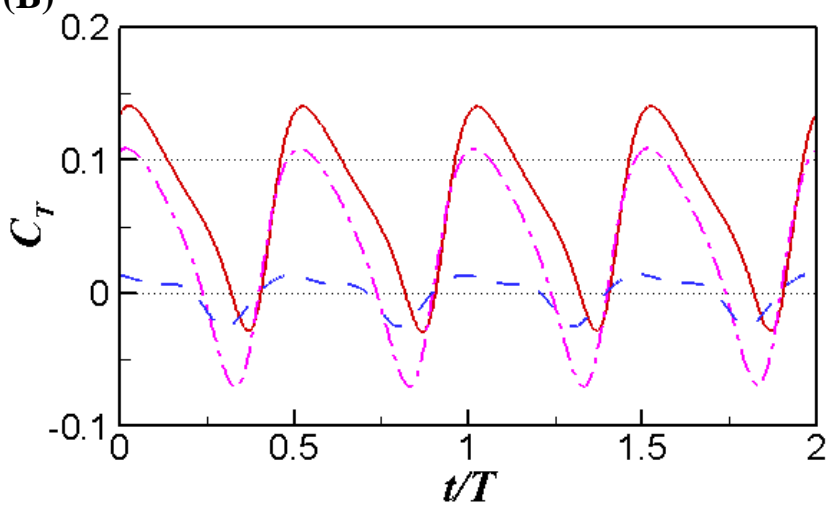

(D)

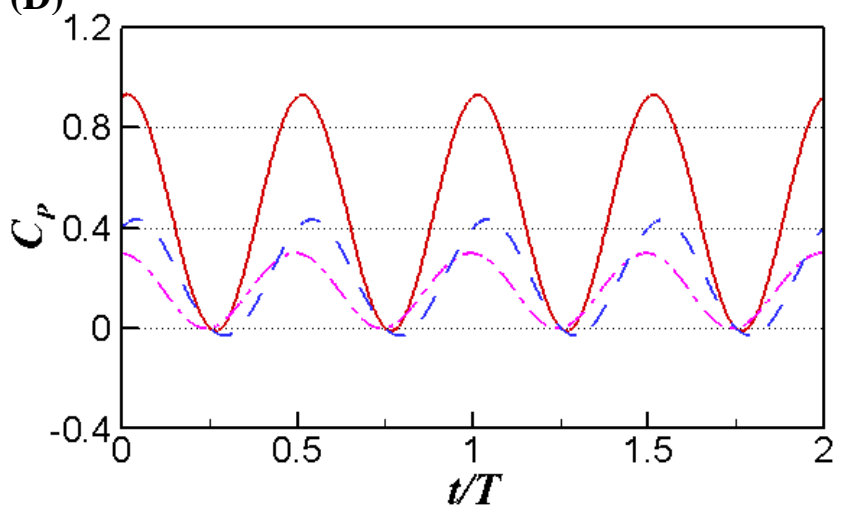

Fig. 8 Instantaneous thrust (CT) and power input coefficient (CP) for fin I (red solid line), fin II (pink dash-dot line) and fin III (blue dash line), and for flexible cases (a, c) and highly flexible cases (b, d). 


\section{RESULTS}

In present study, we focus on the effects of different deformations on the propulsion performance of the caudal fin. Thus, we examined three different cases, as shown in Fig. 7. In the first case (referred as fin I), all springs are in the middle of the fin, while in the second case (referred as fin II), the springs are distributed at the tips. In the third case (referred as fin III), the springs are distributed asymmetrically. In order to measure the rigidity of the ray and fin, we further define three parameters: mass ratio $M^{*}=\rho_{\mathrm{s}} h / \rho_{\mathrm{f}} L$ ( $L$ is the span-wise length of the fin), dimensionless fin rigidity $K_{\mathrm{B}}=E I / \rho_{\mathrm{f}} U_{0}^{2} L^{3}$, and dimensionless ray stiffness $K_{\mathrm{S}}=K / \rho_{\mathrm{f}} U_{0}{ }^{2} \quad(K$ is the spring stiffness). In present work, we choose $M^{*}=0.01, K_{\mathrm{B}}=0.5$ in order to obtain desired deformations. Two different spring stiffness values are used, denoted as flexible $\left(K_{\mathrm{S}}=2 \times 10^{4}\right)$ and highly flexible $\left(K_{\mathrm{S}}=5 \times 10^{3}\right)$ respectively.

Table 2 summarizes the mean thrust coefficient, power input coefficient and efficiency of three fins. It can be seen that the performance of the fin is highly affected by the distribution and flexibility of the fin rays. Fin I has the highest mean thrust and mean power input among all fins in both flexible and highly flexible cases. By increasing the ray's flexibility, both $C_{T}$ and $C_{P}$ increase slightly, while the efficiency has a small decrease. For fin II, it has the highest efficiency due to the lowest power expenditure and relatively high thrust forces. Besides, both the thrust and efficiency rise with the increase of ray's flexibility. In terms of fin III, the thrust force as well as power input is both high, leading to a moderate efficiency. It is interesting to note that when the flexibility of the ray increases to a certain value, the fin generates no thrust, causing a negative efficiency.

Figure 8 demonstrates the time history of the thrust and power input coefficients for three fins with different ray rigidities. It can be seen that both $C_{T}$ and $C_{P}$ vary periodically and have two peaks within one period. For both flexible and highly flexible cases, fin I not only generates the highest peaks in $C_{T}$, but also needs the most power input, leading to moderate propulsion efficiency. This can be explained by its deformation and the fluid field, which will be shown later on. It is seen that for flexible case, fin II and fin III have similar peaks in $C_{T}$, but fin II has lower and wider trough, which leads to lower thrust force. Compared with the other two fins, fin II has the lowest and narrowest peak in $C_{P}$, which explains the reason why it has the highest propulsion efficiency. When the rays become more flexible, the peaks in $C_{T}$ of fin $I$ become slightly higher but narrower, resulting in little increase in thrust generation, whereas an obvious ascent is observed in $C_{P}$ of fin $\mathrm{I}$, which causes the reduction of efficiency as shown in Table 2. Similar trend is observed for fin $I I$ that both the peaks in $C_{T}$ and $C_{P}$ have a tiny increase, but resulting in higher propulsion efficiency. On contrary, both thrust and power expenditure drop rapidly for fin III, and it generates more drag rather than thrust, leading to negative propulsion efficiency.

The deformation patterns of the flexible fins and the $y$ displacements of reference and controlling points are shown in Fig. 9. It can be seen that various ray distributions generate their specific fin deformations. The deformations of fin $I$ and fin II are quite similar to each other, both of which have 'cup' shapes. Besides, they are symmetric due to the symmetry of ray's distribution. However, apparent difference is also noted. For example, for fin I, the central part of the fin leads the tips while the tips lead the middle part for fin II, which is supposed to have effects on the formation of both tip vortices and the wake. Additionally, fin I has much larger effective flapping amplitude than fin II, which is regarded as the reason that fin I generates larger thrust force. As for fin III, instead of having a symmetric 'cup' shape, it deforms asymmetrically due to the asymmetry of ray's distribution.
(A1)

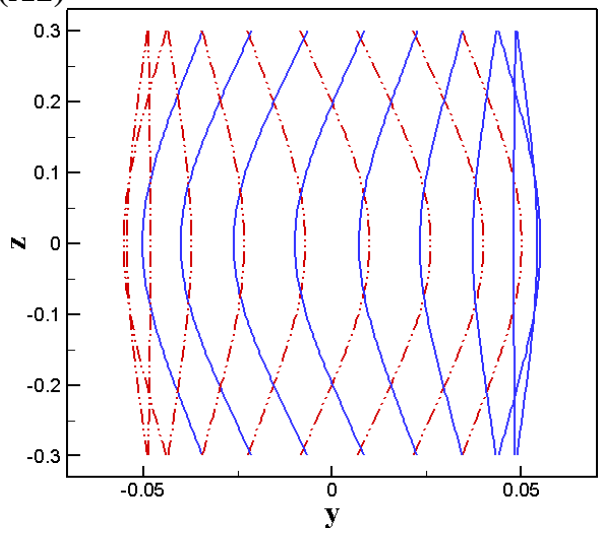

(A2)

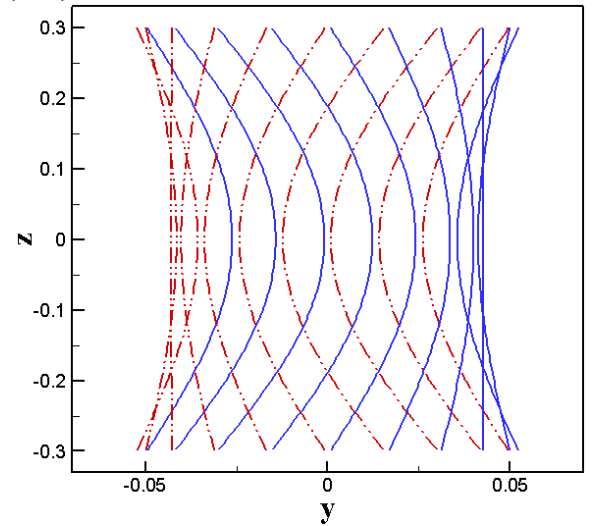

(A3)

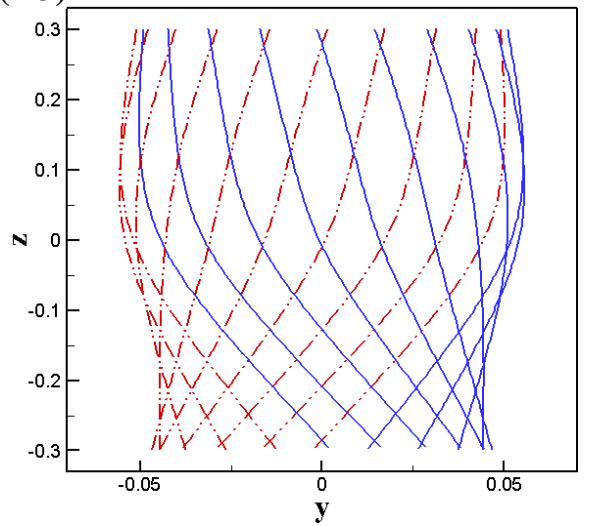

Fig. 9 The fin deformation patterns of fin I (A1), fin II (A2) and fin III (A3), flexible case. The red dash-dot-dot lines and blue solid lines represent fin deformations when the reference points are moving to right and left respectively. 
(A)

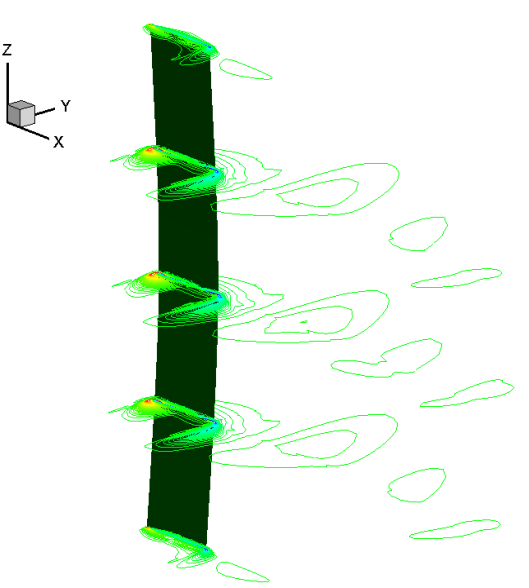

(C)

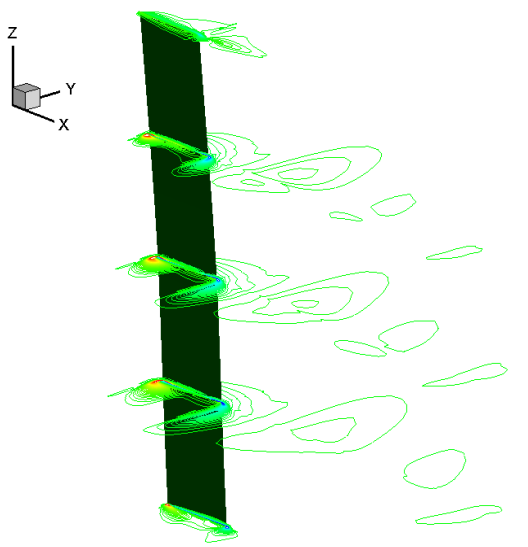

(B)

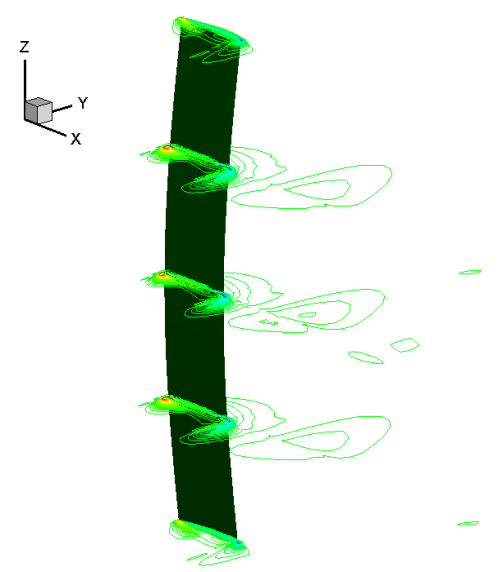

(D)

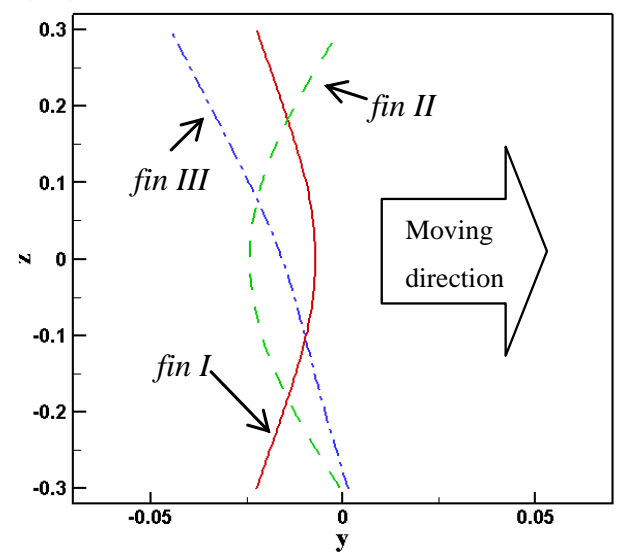

Fig. 10 Flow z-vorticity of fin I(A), fin II (B), fin III (C) at representative slices and schematic illustration of corresponding fin deformations (D), $t=0$, flexible case.

Figure 10 shows the flow vorticity at five different slices as well as the diagram of fin deformations. It can be seen that the vorticity patterns generated by three fins are quite similar. The vortices generated at the tips are weaker than those in the middle. However, the leading edge vortices (LEVs) of fin I are much stronger than those of fin II, which is attributed to the larger effective amplitude as illustrated in Fig. 9. Stronger LEVs induce larger lateral force which is one reason that fin I has higher power expenditure. Another reason is that the rays are distributed in the central region of the fin, where stronger vortices are generated. In contrary, the rays of fin II are distributed at tips where much smaller lateral forces are experienced by the fin, thus leading to higher propulsion efficiency. A closer inspection on the trailing edge vortices (TEVs) reveals that the TEVs of fin I are much stronger than those of fin II, which is responsible for higher thrust generation of fin I. As for fin III, it is observed that the vorticity pattern is asymmetric due to the asymmetrically distributed fin rays. It is seen that the vortices generated at the upper part of the fin are much weaker than those in the lower part, which is responsible for the relatively smaller thrust.

\section{CONCLUSIONS}

This paper investigates the propulsion performance of a three-dimensional flexible caudal fin which is deformable only in span-wise direction. By representing the fin rays via linear springs, the effects of fin deformation on the performance of the fin using two different spring stiffness are studied. The different fin deformations are achieved by various distributions of fin rays. It is found that both the distribution and flexibility of the fin ray have significant impact on the performance of the caudal fin. Under the parameters studied in this paper, the fin I obtains the highest thrust which can be attributed to larger effective heaving amplitude, which leads to a larger effective Strouhal number. Due to smaller lateral forces and ray distribution at tips, fin II consumes the lowest power expenditure, resulting in the highest propulsion efficiency.

Because only two ray rigidities have been studied at present, the details on how the flexibility of the ray affects the propulsion performance still remain unclear. More studies are necessary in order to clarify its effect. 


\section{ACKNOWLEDGMENTS}

Results were obtained using the EPSRC funded ARCHIEWeSt High Performance Computer (www.archie-west.ac.uk ). EPSRC grant no. EP/K000586/1. The first author would like to thank China Scholarship Council (CSC) for the financial support during his study in the UK.

\section{REFERENCES}

Cleaver, D. J., Gursul, I., Calderon, D. E. and Wang, Z., 2014, "Thrust Enhancement Due to Flexible Trailing-edge of Plunging Foils," Journal of Fluids and Structures, 51, 401-412.

Dettmer W and Peric D., 2006, “A Computational Framework for Fluid-structure Interaction: Finite Element Formulation and Applications," Computer Methods in Applied Mechanics and Engineering, 195, 5754-79.

Farhat, C. and Lesoinne, M., 2000, “Two Efficient Staggered Algorithms for the Serial and Parallel Solution of Threedimensional Nonlinear Transient Aeroelastic Problems," Computer Methods in Applied Mechanics and Engineering, 182, 499-515.

Goura, G. S. L., Badcock, K. J., Woodgate, M. A., and Richards, B. E., 2001, "Transformation Methods for the Time Marching Analysis of Flutter," AIAA Paper, 2001-2457.

Habchi, C., Russeil, S., Bougeard, D., Harion, J., Lemenand, T., Ghanem, A., Valle, D. D. and Peerhossaini, H., 2013, "Partitioned Solver for Strongly Coupled Fluid-structure Interaction," Computers and Fluids, 71, 306-319.

Jameson, A., Schmidt, W., and Turkel, E., 1981, "Numerical Solutions of the Euler Equations by Finite Volume Methods Using Runge-Kutta Time-Stepping Schemes," AIAA Paper, 811259.

Jameson, A. 1991, “Time Dependent Calculations Using Multigrid, with Applications to Unsteady Flows Past Airfoils and Wings," AIAA Paper, 81-1259.

Kassiotis C., Ibrahimbegovic A., Niekamp R. and Matthies H., 2011, "Nonlinear Fluid-structure Interaction Problem. Part I: Implicit Partitioned Algorithm, Nonlinear Stability Proof and Validation Examples," Computational Mechanics, 47, 305-23.

Liu, F. and Ji, S., 1996, "Unsteady Flow Calculations with a Multigrid Navier-Stokes Method," AIAA Journal, 34(10), 2047-2053.

Liu, F. and Zheng, X., 1996, "A Strongly-coupled Timemarching Method for Solving the Navier-Stokes and $k-\omega$ Turbulence Model Equations with Multigrid,' Journal of Computational Physics, 128, 289-300.
Liu, W., Xiao, Q. and Cheng, F., 2013, "A Bio-inspired Study on Tidal Energy Extraction with Flexible Flapping Wings," Bioinspiration and Biomimetics, 8(3), 036011.

Liu, W., Xiao, Q. and Zhu, Q., 2016, "Passive Flexibility Effect on Oscillating Foil Energy Harvester," AIAA Journal, 54(4), $1172-1187$

Matthies, H. G., Steindorf, J., 2003, "Partitioned Strong Coupling Algorithms for Fluid-structure Interaction," Computers and Structures, 81(8-11), 805-12.

Shoele, K. and Zhu, Q., 2009, "Fluid-structure Interactions of Skeleton-reinforced Fins: Performance Analysis of a Paired Fin in Lift-based Propulsion," Journal of Experimental Biology, 212, 2679-2690.

Shoele, K. and Zhu, Q., 2010, "Numerical Simulation of a Pectoral Fin During Labriform Swimming," Journal of Experimental Biology, 213, 2038-2047.

Shoele, K. and Zhu, Q., 2012, "Leading Edge Strengthening and the Propulsion Performance of Flexible Ray Fins," Journal of Fluid Mechanics, 693, 402-432.

Tangorra, J. L., Davidson, S. N., Hunter, I. W., Madden, P. G. A., Lauder, G. V., Dong, H., Bozkurttas, $M$ and Mittal, R., 2007, "The Development of a Biologically Inspired Propulsor for Unmanned Underwater Vehicles," IEEE Journal of Oceanic Engineering, 32, 533-550.

Tangorra, J. L., Lauder, G. V., Hunter, I. W., Mittal, R., Madden, P. G. A. and Bozkurttas, M., 2010, "The Effect of Fin Ray Flexural Rigidity on the Propulsive Forces Generated by a Biorobotic Fish Pectoral Fin," Journal of Experimental Biology, 213, 4043-4054.

Wood, C., Gil, A., Hassan, O. and Bonet, J., 2010, "Partitioned Block-Gauss-Seidel Coupling for Dynamic Fluid-structure Interaction," Computers and Structures, 88(23-24), 1367-82.

Xiao, Q. and Liao, W., 2010, "Numerical Investigation of Angle of Attack Profile on Propulsion Performance of an Oscillating Foil," Computers and Fluids, 39(8), 1366-1380.

Xiao, Q., Liao, W., Yang, S. and Peng, Y., 2012, "How Motion Trajectory Affects Energy Extraction Performance of a Biomimic Energy Generator with an Oscillating Foil?" Renewable Energy, 37(1), 61-75.

Zhu, Q. and Shoele, K., 2008, "Propulsion Performance of a Skeleton-strengthened Fin," Journal of Experimental Biology, 211, 2087-2100. 